\title{
Auswertung von Studien zur Prävalenz kindlicher Atopien in Deutschland
}

\author{
W. Hellmeier \\ Landesinstitut für den Öffentlichen Gesundheitsdienst NRW, Bielefeld
}

Viele Indikatoren sprechen dafür, daß allergische Erkrankungen, insbesondere bei Kindern, immer häufiger werden. Epidemiologische Studien, die den Anstieg mit repräsentativen Daten untermauern, liegen allerdings nicht vor. Durch eine gemeinsame Auswertung regionaler Studien $\mathrm{zu}$ Atopien bei Kindern soll untersucht werden, ob ein zeitlicher Trend für Deutschland bestätigt werden kann.

Es werden Studien analysiert, die Angaben zur Prävalenz atopischer Symptome für Kinder eines Altersjahrgangs in jeweils klar definierten Regionen Deutschlands enthalten.
Recherchen erfolgten in MEDLINE und SOMED und wurden durch die Bearbeitung von Literaturverzeichnissen ergänzt. Es wurden zehn Studien aus den Jahren 1987-1994 gefunden, aus denen 24 Beiträge zur Asthmaprävalenz, 21 zur Ekzemprävalenz und 26 zur Häufigkeit allergischer Rhinitis extrahiert werden konnten. Die Diagnosen wurden getrennt analysiert. Nach einer Berechnung der Meta-Schätzer unter Annahme fester bzw. zufälliger Effekte wurden die Einflüsse des Studienjahres und einiger Kovariablen überprüft.
Die Prävalenzschätzungen aus den einzelnen Studien sind sehr unterschiedlich: Die Metaanalysen unter der Annahme zufälliger Effekte berechnen 11,3\% für die Ekzemprävalenz, 3,8\% für die Asthmaprävalenz und 6,3\% für allergische Rhinitis. Aufgrund der hohen Heterogenität sind diese Werte allerdings nicht verallgemeinerbar. - Ein ansteigender Trend läßt sich für keine der drei Diagnosen nachweisen. Variationen der jeweiligen Prävalenzen ergeben sich durch das Alter, die Region, Stadt-Land-Unterschiede und die Berücksichtigung bzw. den Ausschluß von Ausländern in der Stichprobe.

\section{Ausgewählte allergologische Befunde der Bitterfeld-Studie}

\author{
J. Heinrich, H.-E. Wichmann \\ GSF-Forschungszentrum für Umwelt und Gesundheit, Institut für Epidemiologie, Neuherberg
}

Nach der Wiedervereinigung Deutschlands im Jahr 1990 konnten erstmals epidemiologische Studien zur Prävalenz atopischer Erkrankungen durchgeführt werden, die ostdeutsche und westdeutsche Populationen direkt miteinander verglichen. Die Erkenntnisse dieser Studie zeigten höhere Prävalenzraten der allergischen Rhinitis und der allergischen Sensibilisierung in Westdeutschland im Vergleich zu Ostdeutschland sowohl bei Kindern als auch bei jüngeren Erwachsenen (2).

Die Gründe für die größere Verbreitung atopischer Erkrankungen in Westdeutschland sind noch weitgehend unbekannt. Es wird spekuliert, daß
Unterschiede in den Lebensstil- oder Umweltfaktoren das höhere Atopierisiko in Westdeutschland verursacht haben. Beispiele für solche Faktoren sind verkehrsabhängige Außenluftschadstoffe, Innenraumschadstoffe, Zugang $\mathrm{zu}$ Kinderkrippenerziehung ab frühestem Alter, seltenere und leichtere infektiöse Erkrankungen in der frühen Kindheit etc. (2). Wenn einige dieser Lebensstil- und Umweltfaktoren tatsächlich die höheren Atopieraten in den letzten beiden Jahrzehnten in Westdeutschland verursacht haben sollten, dann wäre ein Anstieg der Häufigkeit der Atopie in Ostdeutschland zu erwarten, weil westlicher Lebensstil von der ostdeutschen Bevölkerung angenommen wird und sich die Schadstoffe in Ostdeutschland denen in Westdeutschland angeglichen haben. Es wird untersucht, ob es bereits innerhalb von fünf Jahren nach der Wiedervereinigung zu einem Anstieg der allergischen Sensibilisierung bei Kindern aus Ostdeutschland gekommen ist.

Die Untersuchungsbefunde entstammen dem 1. und 2. Survey der Bitterfeld-Studie (1). Es wurden Kinder aus drei Regionen Sachsen-Anhalts ausgewählt. Zwei unabhängige Stichproben von Kindern im Alter von 5 bis 7 Jahren wurden im Abstand von 3 Jahren in den Jahren 1992/93 und 1995/96 untersucht.

Die Eltern der Kinder wurden gebeten, einen Fragebogen u.a. zu allergischen Erkrankungen und Symptomen des Kindes und in der Familie 